01

\title{
Спектры комбинационного рассеяния н-бутана, изобутана, н-пентана и изопентана в среде метана
}

\author{
(ㄷ Д.В. Петров ${ }^{1,2}$, И.И. Матросов ${ }^{1}$, А.Р. Зарипов ${ }^{1}$ \\ ${ }^{1}$ Институт мониторинга климатических и экологических систем Сибирского отделения РАН, \\ 634055 Томск, Россия \\ ${ }^{2}$ Национальный исследовательский Томский государственный университет, \\ 634050 Томск, Россия \\ e-mail: dmvitpetrov@gmail.com
}

Поступила в редакцию 29.01.2018 г.

В окончательной редакции 02.03.2018 г.

Исследованы особенности спектров комбинационного рассеяния (КР) газообразных $\boldsymbol{H}$-бутана, изобутана, $H$-пентана и изопентана при их нахождении в среде метана при давлении $25 \mathrm{~atm}$. Установлено, что в данных условиях изменение конформационных равновесий данных молекул пренебрежимо мало, а наиболее значимые изменения в спектрах проявляются в диапазоне $2850-3000 \mathrm{~cm}^{-1}$, где имеет место сдвиг полос, соответствующих валентным колебаниям групп $\mathrm{CH}_{2}$ и $\mathrm{CH}_{3}$, в область меньших частот. Полученные данные будут востребованы при определении состава природного газа с помощью спектроскопии КР.

DOI: $10.21883 / O S .2018 .07 .46258 .26-18$

\section{Введение}

Природный газ (ПГ) представляет собой многокомпонентную среду, преимущественно состоящую из метана, его гомологов (этан, пропан, бутаны, пентаны ит.д.) и неуглеводородных соединений, таких как азот, водород и диоксид углерода. Несмотря на широкое распространение газовых хроматографов для определения состава ПГ, достаточно многообещающим на сегодняшний день выглядит применение газоанализаторов, основанных на спектроскопии комбинационного рассеяния (КР) света [1-8]. Основными преимуществами КР газоанализаторов являются высокая скорость измерений, отсутствие расходных материалов, а также возможность одновременного контроля всех молекулярных составляющих газовой среды, концентрация которых превышает порог чувствительности аппаратуры.

Ввиду значительного перекрывания спектров КР различных углеводородов для определения количественного состава ПГ наиболее эффективным является разложение его зарегистрированного спектра КР на спектры КР отдельных компонентов [1]. В связи с этим достоверность данного метода газоанализа напрямую зависит от соответствия спектров КР компонентов ПГ, являющихся базисными, тем, которые находятся непосредственно в ПГ. Ранее было установлено [9,10], что определение состава ПГ целесообразно проводить при фиксированных значениях давления и температуры ввиду того, что данные параметры оказывают значительное влияние на спектр КР метана, который является доминирующим компонентом ПГ. Помимо этого было исследовано, как этан, пропан, азот, водород и диоксид углерода влияют на спектр метана, а метан влияет на их спектры [11-13].

Настоящая работа является продолжением цитированных работ и посвящена исследованию спектров КР га- зообразных $\boldsymbol{H}$-бутана, изобутана (метилпропана), $\boldsymbol{H}$-пентана и изопентана (2-метилбутана), находящихся в среде метана при давлении $25 \mathrm{~atm}$.

\section{Экспериментальная часть}

Исследования были выполнены на экспериментальной установке, построенной на КР-спектрометре, ранее использованном в работе [1]. Для возбуждения КР применялся непрерывный $\mathrm{Nd}$ : YAG-лазер с выходной мощностью $2 \mathrm{~W}$ на длине волны $532 \mathrm{~nm}$. Рассеянное излучение собиралось в направлении, перпендикулярном направлению распространения лазерного излучения, с помощью пары идентичных линзовых объективов с относительным отверстием $f / 1.8$ и фокусным расстоянием $50 \mathrm{~mm}$. Для разложения собранного излучения в спектр использовался специализированный спектральный прибор MKR-2 с входным относительным отверстием $f / 1.8$. Регистрация спектров проводилась с помощью ПЗС-матрицы Hamamatsu S10141 $(2048 \times 256$ ріх $)$ с охлаждением Пельтье до $-10^{\circ} \mathrm{C}$, работающей в режиме ПЗС-линейки за счет вертикального биннинга зарядов. С данной матрицей при входной щели шириной $40 \mu \mathrm{m}$ и дифракционной решетке 1600 grooves $/ \mathrm{mm}$ обеспечивалось разрешение $\sim 6 \mathrm{~cm}^{-1}$ при дисперсии $1.8 \mathrm{~cm}^{-1} /$ ріх и одновременной регистрации спектрального диапазона $200-3800 \mathrm{~cm}^{-1}$. Частотная калибровка спектрометра была проведена по спектру излучения неоновой лампы [14].

На данном оборудовании были зарегистрированы спектры КР газообразных $н$-бутана и изобутана при давлениях $1 \mathrm{~atm}$, а также $\boldsymbol{H}$-пентана и изопентана при давлениях $0.5 \mathrm{~atm}$. После регистрации спектра каждого газа в кювету добавлялся метан до достижения суммарного давления $25 \mathrm{~atm}$ и производилась регистрация 
спектра данной смеси. Таким образом, концентрация бутанов в смесях составляла $\sim 4 \%$, пентанов $\sim 2 \%$, что соответствует максимальным значениям концентраций данных газов в ПГ [15]. Каждый полученный спектр представлял собой сумму 100 кадров, при этом время экспозиции одного кадра в серии с бутанами составляло $1 \mathrm{~s}$, с пентанами - $10 \mathrm{~s}$ ввиду меньшей концентрации (и соответственно сигналов КР) последних. Для проведения анализа изменений спектров исследуемых молекул также было получено 2 спектра КР чистого метана при давлении 25 atm с эквивалентными временами экспозиции. Давление исследуемых газовых сред контролировалось манометром с погрешностью $<0.02 \mathrm{~atm}$. Чистота используемых газов > 99.9\%. Для устранения нежелательных температурных эффектов газовая кювета была термостабилизирована на $300 \pm 1 \mathrm{~K}$.

\section{Результаты и их обсуждение}

Наиболее интенсивные полосы всех исследуемых молекул расположены в области 2850-3000 $\mathrm{cm}^{-1}$ (рис. 1). В случае $н$-бутана и пентанов в данной части спектров находятся полосы, соответствующие валентным колебаниям групп $\mathrm{CH}_{2}$ и $\mathrm{CH}_{3}$, которые условно можно разделить на следующие участки: $2860-2880 \mathrm{~cm}^{-1}$ симметричные $\mathrm{CH}_{2}, 2880-2910 \mathrm{~cm}^{-1}$ - симметричные $\mathrm{CH}_{3}, 2910-2930 \mathrm{~cm}^{-1}$ - антисимметричные $\mathrm{CH}_{2}$, 2940-2980 $\mathrm{cm}^{-1}$ - антисимметричные $\mathrm{CH}_{3}$ [16-20]. В отличие от данных молекул в случае изобутана, в котором отсутствует группа $\mathrm{CH}_{2}$, полоса с частотным сдвигом $2880 \mathrm{~cm}^{-1}$ принадлежит валентному колебанию $\mathrm{C}-\mathrm{H}$, а валентные симметричные колебания группы $\mathrm{CH}_{3}$ располагаются в диапазоне 2890-2930 $\mathrm{cm}^{-1}[18,20]$.

Другая группа полос, интенсивности которых более чем на порядок слабее, находится в области $750-1600 \mathrm{~cm}^{-1}$. В данной части спектров располагаются полосы, соответствующие валентным колебаниям $\mathrm{C}-\mathrm{C}$, а также деформационным колебаниям групп $\mathrm{CH}_{2}$ и $\mathrm{CH}_{3}$. В свою очередь, наблюдаемые в диапазоне $2600-2800 \mathrm{~cm}^{-1}$ полосы принадлежат их обертонам и комбинационным модам, а их относительно высокие интенсивности обусловлены наличием резонансов Ферми с валентными колебаниями $\mathrm{CH}_{2}$ и $\mathrm{CH}_{3}$.

Также во всех полученных спектрах в диапазоне $300-500 \mathrm{~cm}^{-1}$ наблюдались характерные полосы, соответствующие ножничным колебаниям C-C-C. Отметим, что поскольку данные полосы были на краю регистрируемого диапазона, то их интенсивность была несколько ослаблена ввиду специфики используемого спектрального прибора.

Важной особенностью данных молекул является то, что они, за исключением изобутана, имеют конформационные изомеры, которые различаются расположением атомов в пространстве ввиду поворотов вокруг связи С-С. Так, н-бутан и изопентан имеют по два стабильных конформера (транс- и гош-), а н-пентан -
Полосы КР н-бутана, соответствующие различным конформерам

\begin{tabular}{c|c|c}
\hline $\begin{array}{c}\text { Частотный } \\
\text { сдвиг, } \mathrm{cm}^{-1}\end{array}$ & $\begin{array}{c}\text { Тип конформера } \\
{[17,22]}\end{array}$ & Тип колебаний \\
\hline 319 & гош & ножничные C-C-C \\
430 & транс & \\
\hline 831 & гош & валентные C-C \\
838 & транс & \\
\hline 1060 & гонс & валентные C-C \\
1077 & &
\end{tabular}

три (транс-транс-, транс-гош- и гош-гош-). В газовой среде все данные конформационные изомеры присутствуют одновременно, при этом поскольку энергия транс-конформеров ниже, чем гош-конформеров, их относительная концентрация при комнатной температуре выше. Очевидно, что ввиду различного строения конформеров их спектры КР несколько различаются. Данные различия в первую очередь проявляются в полосах, соответствующих валентным колебаниям C-C и ножничным колебаниям $\mathrm{C}-\mathrm{C}-\mathrm{C}$. В спектре КР н-бутана присутствуют три пары таких полос (таблица), которые были ранее $[21,22]$ использованы для изучения изменения конформационного равновесия при изменении температуры. В полученных спектрах КР пентанов ввиду большего количества разрешенных колебаний полосы различных конформеров $[19,20]$ сильно перекрываются, и выделить в явном виде аналогичные характерные полосы достаточно трудно. Тем не менее в случае изменения конформационного равновесия таких углеводородов будет иметь место перераспределение интенсивностей и изменение контуров наблюдаемых полос КР. В свою очередь, поскольку увеличение давления также может быть причиной конформационных переходов [23-25], то оценка их значимости в исследуемых условиях также представляет интерес.

Для определения изменений спектров КР исследуемых молекул в среде метана была проведена процедура разложения полученных спектров КР бинарных смесей на спектры КР чистых компонентов. Вклад каждого компонента в спектр смеси определялся с помощью методики, описанной в [1]. Согласно полученным после выполнения данной процедуры разностным спектрам, приведенным на рис. 1, можно заключить, что в спектрах КР смесей имеют место изменения спектральных характеристик как исследуемых молекул, так и метана. В частности, наблюдается уширение его полос $v_{2}$ и $v_{3}$, a также сдвиг полосы $v_{1}$ в область меньших частот. Отметим, что ввиду больших экспозиций спектров в серии с пентанами наиболее интенсивная полоса метана $v_{1}\left(2917 \mathrm{~cm}^{-1}\right)$ находилась в насыщении. Поэтому в полученных разностных спектрах (рис. $1, c, d$ ) в месте ее расположения имеются неинформативные участки, 

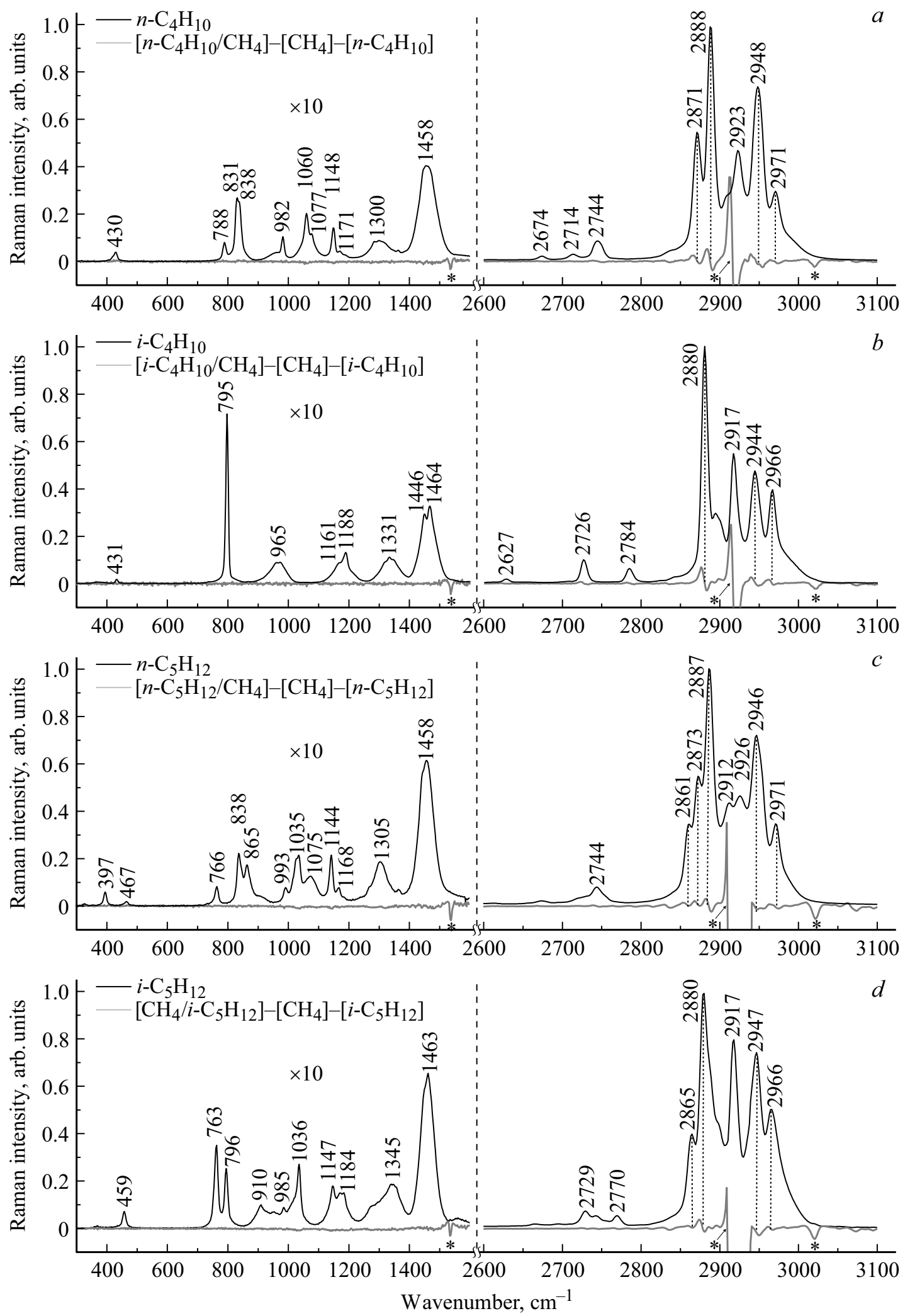

Рис. 1. Спектры КР н-бутана $(a)$, изобутана $(b), H$-пентана $(c)$, изопентана $(d)$ и результаты изменения спектров КР их смесей с метаном по сравнению со спектрами чистых компонентов. Интенсивности полос в диапазоне $300-1600 \mathrm{~cm}^{-1}$ увеличены в 10 раз. Звездочками отмечены положения полос КР метана $v_{1}, v_{2}$ и $v_{3}$. 

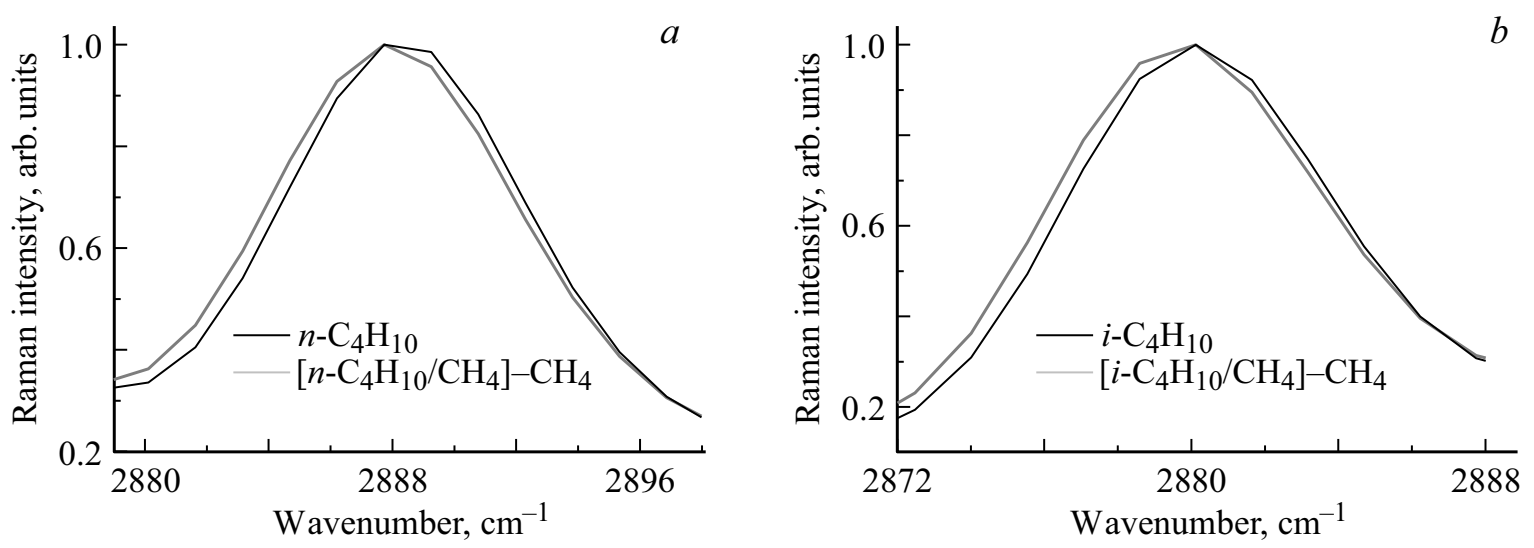

Рис. 2. Наиболее интенсивные полосы КР $н$-бутана $(a)$ и изобутана $(b)$, зарегистрированные при давлении $1 \mathrm{~atm}$ и в среде метана при давлении $25 \mathrm{~atm}$.

которые не позволяют оценить изменения спектров в диапазоне $\sim 2910-2940 \mathrm{~cm}^{-1}$. Отметим, что наблюдаемые изменения спектральных характеристик полос метана согласуются с данными, представленными в работе [11].

В свою очередь, изменения спектров КР бутанов и пентанов наблюдаются только в области $2850-3000 \mathrm{~cm}^{-1}$, где имеет место сдвиг всех интенсивных полос данных молекул в область меньших частот при нахождении в среде метана. Оценка данных смещений была проведена на примерах наиболее интенсивных полос бутанов (рис. 2) посредством их аппроксимации гауссовыми контурами. В результате данной процедуры было установлено, что частотные сдвиги полос в спектрах чистых компонентов составляют 2888.4 и $2880.2 \mathrm{~cm}^{-1}$, а в среде метана 2888.0 и $2879.9 \mathrm{~cm}^{-1}$ для н-бутана и изобутана соответственно. Среднеквадратическое отклонение при аппроксимации не превышало $0.1 \mathrm{~cm}^{-1}$. Таким образом, судя по виду полученных разностных спектров, наблюдаемые сдвиги во всех спектрах не превышали $0.4 \mathrm{~cm}^{-1}$. Изменения полуширин данных полос, а также изменения какихлибо спектральных характеристик полос, расположенных в области $300-1600 \mathrm{~cm}^{-1}$, были пренебрежимо малы. Аналогичное поведение полос, соответствующих различным колебаниям, было отмечено при исследовании метан-этановых смесей [13]. Крайне слабое изменение контуров полос в диапазоне $300-1600 \mathrm{~cm}^{-1}$ также свидетельствует о том, что в анализируемых смесях изменений конформационных равновесий пентанов и $\boldsymbol{H}$ бутана зарегистрировано не было. Таким образом, с учетом интенсивности полос в данном диапазоне и значения шума можно утверждать, что изменение относительных концентраций конформеров не превышало $1 \%$. Такая относительная погрешность при определении содержания данных молекул в составе ПГ является достаточно малой, в связи с чем эффектами изменения конформационных равновесий можно пренебречь.

\section{Заключение}

Установлено, что по сравнению со спектрами КР газообразных $\boldsymbol{\mu}$-бутана и изобутана при давлении $1 \mathrm{~atm}$, а также $t$-пентана и изопентана при давлении $0.5 \mathrm{~atm}$ в спектрах КР данных молекул в среде метана при давлении $25 \mathrm{~atm}$ полосы валентных колебаний групп $\mathrm{CH}_{2}$ и $\mathrm{CH}_{3}$, расположенные в области $2850-3000 \mathrm{~cm}^{-1}$, сдвигаются в область меньших частот. В свою очередь, значимых изменений спектральных характеристик полос, расположенных в области $300-1600 \mathrm{~cm}^{-1}$, которые соответствуют валентным колебаниям $\mathrm{C}-\mathrm{C}$, a также деформационным колебаниям групп $\mathrm{CH}_{2}, \mathrm{CH}_{3}$ и $\mathrm{C}-\mathrm{C}-\mathrm{C}$, не наблюдалось. Данный факт свидетельствует о том, что изменение конформационных равновесий $\boldsymbol{\mu}$-бутана, $\boldsymbol{H}$-пентана и изопентана в данных условиях пренебрежимо мало.

Полученные данные позволяют сделать вывод о том, что для определения концентрации исследуемых молекул в составе ПГ с помощью спектроскопии КР наиболее удобным является диапазон $300-1600 \mathrm{~cm}^{-1}$ ввиду отсутствия необходимости применения поправок на их спектры КР.

Работа выполнена при поддержке РФФИ в рамках научного проекта № 16-32-60111 мол_а_дк.

\section{Список литературы}

[1] Petrov D.V., Matrosov I.I. // Appl. Spectrosc. 2016. V. 70. N 10. P. 1770. doi 10.1177/0003702816644611

[2] Петров Д.В., Матросов И.И., Тихомиров А.А. // ЖПС. 2015. T. 82. № 1. C. 124; Petrov D.V., Matrosov I.I., Tikhomirov A.A. // J. Appl. Spectrosc. 2015. V. 82. N 1. P. 120. doi 10.1007/s10812-015-0073-4

[3] Булдаков М.А., Королев Б.В., Корольков В.А., Матросов И.И., Петров Д.В., Тихомиров А.А. // Оптический журнал. 2013. Т. 80. № 7. С. 27; Buldakov M.A., Korolev B.V., Korolkov V.A., Matrosov I.I., Petrov D.V., Tikhomirov A.A. // J. Opt. Technol. 2013. V. 80. N 7. P. 426. doi 10.1364/JOT.80.000426 
[4] Kiefer J., Seeger T., Steuer S., Schorsch S., Weikl M.C., Leipertz A. // Meas. Sci. Tech. 2008. V. 19. N 8. P. 085408. doi 10.1088/0957-0233/19/8/085408

[5] Eichmann S.C., Kiefer J., Benz J., Kempf T., Leipertz A., Seeger T. // Meas. Sci. Technol. 2014. V. 25. N 7. P. 075503-1. doi 10.1088/0957-0233/25/7/075503

[6] Buric M.P., Chen K.P., Falk J., Woodruff S.D. // Appl. Opt. 2009. V. 48. N 22. P. 4424. doi 10.1364/AO.48.004424

[7] Hippler M. // Anal. Chem. 2015. V. 87. N 15. P. 7803. doi 10.1021/acs.analchem.5b01462

[8] Sharma R., Poonacha S., Bekal A., Vartak S., Weling A., Tilak V., Mitra C. // Opt. Eng. 2016. V. 55. N 10. P. 104103. doi 10.1117/1.OE.55.10.104103

[9] Петров Д.В. // ЖПС. 2017. Т. 84. № 3. C. 399; Petrov D.V. // J. Appl. Spectrosc. 2017. V. 84. N 3. P. 420. doi 10.1007/s10812-017-0486-3

[10] Petrov D.V. // J. Raman Spectrosc. 2017. V. 48. N 11. P. 1426. doi $10.1002 /$ jrs.5141

[11] Petrov D.V. // Spectrochim. Acta. A. 2018. V. 191. P. 573. doi 10.1016/j.saa.2017.10.058

[12] Петров Д.В., Матросов И.И., Сединкин Д.О., Зарипов A.P. // Опт. и спектр. 2018. Т. 124. № 1. С. 12; Petrov D.V., Matrosov I.I., Sedinkin D.O., Zaripov A.R. // Opt. Spectrosc. 2018. V. 124. N 1. P. 8. doi 10.1134/S0030400X18010137

[13] Petrov D.V. // J. Raman Spectrosc. 2018. doi 10.1002/jrs.5326

[14] Petrov D.V., Matrosov I.I., Sedinkin D.O., Zaripov A.R. // Proc. SPIE. 2017. V. 10466. P. 1046606. doi 10.1117/12.2286319

[15] ГОСТ 31371.7-2008. Газ природный. Определение состава методом газовой хроматографии.

[16] Shimanouchi T. Tables of vibrational molecular frequencies. NSRDS-NBS 39. Washington: National bureau of standards (U.S.), $1972.164 \mathrm{p}$

[17] Murphy W.F., Fernandez-Sanchez J.M., Raghavachari K. // J. Phys. Chem. 1991. V. 95. N 3. P. 1124. doi $10.1021 / \mathrm{j} 100156 \mathrm{a} 020$

[18] Evans J.C., Bernstein H.J. // Can. J. Chem. 1956. V. 34. N 8. P. 1037. doi 10.1139/v56-137

[19] Keefe C.D., Jaspers-Fayer S. // Vib. Spectrosc. 2011. V. 57. N 1. P. 72. doi 10.1016/j.vibspec.2011.05.007

[20] Mirkin N.G., Krimm S. // J. Mol. Str. 2000. V. 550-551. P. 67. doi 10.1016/S0022-2860(00)00513-5

[21] Verma A.L., Murphy W.F., Bernstein H.J. // J. Chem. Phys. 1974. V. 60. N 4. P. 1540. doi $10.1063 / 1.1681228$

[22] Kint S., Scherer J.R., Snyder R.G. // J. Chem. Phys. 1980. V. 73. N 15. P. 2599. doi 10.1063/1.440471

[23] Jorgensen W.L. // J. Am. Chem. Soc. 1981. V. 103. N 16. P. 4721. doi 10.1021/ja00406a010

[24] Kato M., Taniguchi Y. // J. Chem. Phys. 1991. V. 94. N 6. P. 4440. doi 10.1063/1.460740

[25] Whalley E. // Rev. Phys. Chem. Jap. 1980. V. 50. P. 119. 